\title{
Estilo de vida e fatores de risco associados ao aumento da gordura corporal de mulheres
}

\author{
Lifestyle and risk factors associated to body fat increase in women
}

\author{
Carlos Alexandre Fett ${ }^{1}$ \\ Waléria Christiane Rezende Fett ${ }^{2}$ \\ Júlio Sérgio $M$ archini ${ }^{2}$ \\ Rosane Pilot Pessa Ribeiro ${ }^{3}$
}

${ }^{1}$ FaculdadedeEducação Física da Universidade Federal de M ato Grosso. Av. Fernando Correa da Costa s/n, Campus. 78068-900 Cuiabá MT. cafett@hotmail.com ${ }^{2}$ Laboratório de Espectrometria de M assa, Divisão de Nutrologia do Departamento deClínica Médica, Faculdadede M edicina, Universidade de São Paulo.

${ }^{3}$ Escola de Enfermagem de Ribeirão Preto, Departamento de Enfermagem $M$ aternoInfantil eSaúde Pública, Universidade deSão Paulo.
Abstract Theobjectives wereto describethe association between body mass index $\left(\mathrm{BMI}, \mathrm{kg} / \mathrm{m}^{2}\right)$, body composition and risk factors to metabolic diseases; observe the prevalence of metabolic syndrome and list the characteristics of overweight and obese women. Voluntaries $(n=50 ; B M I=31 \pm 6$; age $=36 \pm 11$ years old), were evaluated regarding clinical examination, anthropometrics measurements, samples of blood and urine, resting energy expenditureand food register. Phases in which they becomeobesein descending order: adulthood, pregnancy, adolescence, over 40 years old and after marriage. The odds to have one or more obese family members were $316 \%$. They were anxious $(60 \%)$, depressives $(12 \%)$, compulsives $(34 \%)$ and had sleep disturbance (32\%). The odds to dyslipidemia was $28 \%$, to hypertension was $25 \%$ and to glucose over $100 \mathrm{mg} / \mathrm{dL} 35 \%$. They were in caloric deficit, but, nitrogen balance was positive. The metabolic syndrome was present in $25 \%$ of these women and was positively correlated with body fat indicators and age. The obesity of thesewomen seems to be multifactorial with a family influence that could be caused by genetics and environment contributions. The emotional/physical balance should be influenced on this process.

Key words BM I, M etabolic syndrome, Emotional factors, Resting energy expenditure, Nitrogen balance
Resumo Os objetivos eram descrever a associação entreíndice de massa corporal (IM C, $\left.\mathrm{kg} / \mathrm{m}^{2}\right)$, porcentagem de gordura e fatores de risco para doenças metabólicas; observar a prevalência da síndrome metabólica e relacionar às características de mulheres com sobrepeso e obesas. As voluntárias ( $n=50$; IM C $=31 \pm 6$; idade $=36 \pm 11$ anos) eram avaliadas quanto ao exame clínico, medidas antropométricas, coletas de sanguee urina, gasto energético de repouso e registro alimentar. Fases em que se tornaram obesas em ordem decrescente: idadeadulta, gravidez, adolescência, depois dos 40 anos e depois do casamento. 0 odds deter um ou maisfamiliares obesos era de $316 \%$. Eram ansi osas (60\%), depressivas (12\%), compulsivas (34\%) e com distúrbios do sono (32\%). 0 odds para dislipidemia era $28 \%$, hipertensão, $25 \%$ e para glicemia acima de $100 \mathrm{mg} / \mathrm{dL}$ era de $35 \%$. Elas estavam em déficit calórico, mas o balanço nitrogenado era positivo. A síndromemetabólica era presente em $25 \%$ delas e estava positivamente correlacionada a indicadores da gordura corporal eidade. A obesidadedestas mulheres era multifatorial com influência familiar, podendo ter contribuições genéticas e ambientais. 0 equilíbrio físico/emocional parece ter influenciado neste processo.

Palavras-chave IMC, Síndrome metabólica, Fatores emocionais, Gasto energético de repouso, Balanço nitrogenado 
Introdução

A obesidade é o acúmulo de energia em excesso sob a forma de gordura, pela maior ingestão do que o gasto, influenciada por um complexo de interações genéticas, fatores ambientais e comportamentais ${ }^{1,2}$. Está associada a várias doenças crônicas, como doenças arteriocoronarianas (DAC), hipertensão arterial, diabetes tipo 2, hiperlipidemia, ácido úrico aumentado ${ }^{1}$, câncer, distúrbios do sono ${ }^{3}$ efatores emocionais ${ }^{4}$. É estimado que, em 2020, dois terços do gasto global com doenças serão atribuídos a doenças crônicas não transmissíveis, por falta de exercícios e consumo calórico excessivo ${ }^{5}$.

A disponível oferta de alimentos palatáveis, calóricos e de baixo custo e a pouca atividadefísica da atualidade desencadearam a epidemia mundial de obesidade ${ }^{1}$. Ainda, a proporção dos macronutrientes na al imentação pareceinfluenciar o desenvolvimento da obesidade ${ }^{6,7}$, mas este é um assunto controverso na literatura ${ }^{8,9}$. Ainda, para redução de peso corporal, é necessário estar em bal anço calórico negativo, sendo utilizada a atividade física e a restrição calórica para tal. Entretanto, embora tenhamos acumulado nos últimos trinta anos investimentos e conhecimentos para o seu tratamento, a obesidade aumentou de forma importantenestemesmo período ${ }^{10}$. Além disso, entre 90 e $95 \%$ das pessoas que perdem peso não conseguem mantêlo em longo prazo ${ }^{11}$.

Portanto, a complexa interação entre o estilo de vida e alterações da composição corporal exige uma abordagem que não pode ser reduzida a variáveis puramente quantitativas. A orientação determinista, baseada unicamenteno conceito de balanço energético, tem demonstrado ser ineficienteno controle da obesi dadeem nível populacional, exigindo uma reflexão além desta orientação ${ }^{12}$. Então, investigar os aspectos comportamentais contemplando questões filosóficas e emocionais do obeso e do ato de comer poderá contribuir para um entendimento mais amplo do que o levou a se tornar obeso e, talvez, melhor controlar esta situação.

Então, os principais objetivos deste estudo foram verificar a interação, associação, prevalência e odds da obesidade destas mulheres quanto: 1) à obesidade de seus familiares; 2 ) à fase da vida em que a obesidade se desenvolveu; 3) à proporção dos macronutrientes, total calórico e a quantidadeingerida deleite/ caféingeridos; 4) ao gasto energético de repouso etotal; 5) aos desconfortos físicos e emocionais; e, por último, 6) aos fatores de risco para DAC e para síndrome metabólica.
Metodologia

\section{Voluntárias}

Cinquenta mulheres participaram deste estudo, com idades entre 18 e 64 anos e índice de massa corporal (IM C, kg/ $\mathrm{m}^{2}$ ) entre 25 e 57 ( Tabela 1). 0 recrutamento foi feito pela divulgação de cartazes no campus da Universidade de São Paulo de Ribeirão Preto. 0 estudo foi previamente aprovado pelo Comitê de Ética em Pesquisa do H ospital das Clínicas da Faculdade de M edicina de Ribeirão Preto da Universidade de São Paulo (HCFM RPUSP) e todas assinaram documento deconsentimento livree esclarecido.

\section{Exame clínico}

O exame clínico foi realizado sempre no HCFM RPUSP, pelo mesmo médico da equipe de Nutrologia do Departamento de Clínica M édica. Foram coletadas informações sobre a história médica, antecedente de doenças, obesidade de parentes próximos (pai, mãe e irmão(ã) (s)), exame físico geral e específico, aspectos emocionais, consumo de café e/ou leite, informados pela voluntária ao médico. A pressão arterial foi medida após dez minutos de repouso na posição sentada.

\begin{tabular}{lrrr}
\hline \multicolumn{4}{c}{ Tabela 1. Características gerais das voluntárias $(\mathrm{n}=50)}$. \\
\hline \multicolumn{1}{c}{ Variáveis } & \multicolumn{1}{c}{ M édia } & M ínimo & M áximo \\
& $\pm \mathrm{DP}$ & & \\
\hline Peso $(\mathrm{kg})$ & $83 \pm 16$ & 61 & 142 \\
Altura $(\mathrm{cm})$ & $164 \pm 6$ & 154 & 180 \\
Idade $(\mathrm{anos})$ & 3611 & 18 & 64 \\
IMC $\left(\mathrm{kg} / \mathrm{m}^{2}\right)$ & $31 \pm 6$ & 25 & 57 \\
$\%$ gordura & $41 \pm 6$ & 29 & 50 \\
M assa magra $(\mathrm{kg})$ & $46 \pm 9$ & 37 & 70 \\
Massa gorda $(\mathrm{kg})$ & $34 \pm 12$ & 18 & 71 \\
Circunferência do & $103 \pm 14$ & 84 & 167 \\
abdômen $(\mathrm{cm})$ & & & \\
Colesterol total (mg/dL) & $184 \pm 30$ & 141 & 236 \\
HDL (mg/dL) & 5512 & 36 & 86 \\
LDL (mg/dL) & $108 \pm 27$ & 71 & 166 \\
Triglicérides $(\mathrm{mg} / \mathrm{dL})$ & $105 \pm 52$ & 46 & 343 \\
Ácido úrico $(\mathrm{mg} / \mathrm{dL})$ & $4 \pm 1$ & 2,6 & 8,1 \\
Glicose $(\mathrm{mg} / \mathrm{dL})$ & $93 \pm 10$ & 78 & 119
\end{tabular}




\section{Antropometria}

A massa corporal (precisão de $0,1 \mathrm{~kg}$ ) foi avaliada, pela manhã em jejum, sem sapatos, usando roupão (Filizola $®$ Eletrônica ID 1500). A estatura (precisão de 0,5 cm) foi obtida com a voluntária em pé e descalça, encostada na parede. Foram medidas as pregas cutâneas do tríceps sobre 0 músculo e na parte média entre 0 acrômio e 0 olecrano, da supra-ilíaca dois centímetros acima da crista ilíaca ântero-superior, da coxa entre a articulação do quadril ea patela, sobre o músculo reto femoral ea do abdômen a dois centímetros a direita da cicatriz umbilical. A circunferência do abdômen foi obtida na posição anatômica, paralelamenteao solo, sobrea cicatriz umbilical ea do quadril sobre o trocanter maior (precisão de 0,1 $\mathrm{cm}$ ), com uma fita metálica inextensível ${ }^{13}$. Eram então classificadas a circunferência do abdômen (zona de alerta $=80-88 \mathrm{~cm}$; zona de risco $>88$ $\mathrm{cm})^{14}$ e, o índice abdômen/quadril (IAQ), $(>0,90)^{11}$ para risco de doenças metabólicas.

Coleta de urina e nitrogênio urinário (NU)

Para coleta de urina de 24 horas, era despre zada a primeira urina da manhã e então coletada toda a urina expelida até a primeira da manhã seguinte. A dosagem do NU foi feita pelo método Kjeldahl de 1883, no Laboratório de Espectrometria de M assa da FM RPUSP. O balanço nitrogenado (BN) foi estimado subtraindo o total do nitrogênio ingerido menos o nitrogênio excretado, assumindo-se que cada grama de $\mathrm{N}$ re presenta $6,25 \mathrm{~g}$ de proteína ${ }^{15}$.

\section{Coleta de sangue}

0 sangue foi coletado em jejum de doze horas na rotina do Laboratório Central do Hospital das Clínicas da FM RPUSP. Os triglicérides, HDL-colesterol e colesterol total foram dosados por métodos enzimáticos, seguindo as orientações dos manuais dos cartuchos de reagentes Flex ${ }^{\circledR}$, no Laboratório de Nutrição. O LDL-colesterol foi calculado pela fórmula: $L D L=$ Colesterol total - HDL - triglicérides/5. A glicemia foi dosada pela adaptação do método da Hexokinase-glicose-6-fosfato no Laboratório Central.

\section{Síndrome metabólica}

A síndromemetabólica era classificada quando existiam três ou mais das cinco condições a seguir: circunferência do abdômen $>88 \mathrm{~cm}$; triglicérides $\geq 150 \mathrm{mg} / \mathrm{dL}$; H DL $<50 \mathrm{mg} / \mathrm{dL}$; pressão arterial $\geq 130$ (sistólica)/85 (diastólica) $\mathrm{mmHg}$; glicose de jejum $\geq 110 \mathrm{mg} / \mathrm{dL}^{2}$. Ainda, foi utilizado um escore dos fatores para síndrome metabólica de zero a cinco. Era atribuído zero quando havia ausência de qualquer fator para síndrome metabólica e uma unidade para cada item presente. Este escorefoi utilizado para correlacionar a outros fatores de risco.

\section{Gasto energético de repouso (GER)}

O GER foi medido após jejum de doze horas (V máx. 29 Sensor M edics ${ }^{\circledR}$ ). Elas repousavam por trinta minutos sentadas, trinta minutos deitadas e permaneciam para ser medida a calorimetria por mais trinta minutos. Elas deviam ficar em repouso absoluto, evitando inclusive conversar, mas não era permitido dormir. O GER diário foi calculado pela equação de Weir ${ }^{16}$.

\section{Ingestão alimentar}

As voluntárias foram orientadas por nutricionista do Hospital das Clínicas da FM RPUSP para fazerem o registro alimentar de uma semana. Para conversão das medidas caseiras em gramas dos alimentos ingeridos, foi utilizada a tabela para avaliação de consumo alimentar em medidas caseiras e, em seguida, convertidas para composição química dos alimentos ${ }^{17}$. Estes valores foram utilizados para o cálculo do balanço nitrogenado.

Tratamento estatístico

e avaliação dos resultados

Este foi um estudo observacional do tipo série de casos. Foi calculado o odds para diversas variáveis, dividindo-se a probabilidade, que é 0 número de casos positivos de uma determinada condição, pelo número total de casos menos o número de casos positivos:

odds $=\mathrm{P}:(1-\mathrm{P})$,

sendo $\mathrm{P}$ a probabilidade. A ingestão de leitee/ ou café, consumo e gasto energético e balanço nitrogenado não sofreram tratamento estatístico espeć́fico,sendo apresentado na forma de porcentagem de voluntárias. Para análise da associação do escore dos fatores para síndrome metabólica a outros fatores de risco, foi utilizada a correlação linear de Pearson, a 5\% de significância e intervalo de confiança de $95 \%{ }^{18}$. 


\section{Resultados}

\section{Características gerais}

As principais características das voluntárias estão na Tabela 1. Elas estavam classificadas como obesas tanto pelo IMC quanto pela porcentagem de gordura corporal ${ }^{13}$. Os valores da bioquímica sanguínea estavam em valores considerados normais.

Início da obesidade

Durante o exame clínico, foi investigada a fase da vida mais precoce em que a voluntária se tornou obesa. Não eram computadas na categoria as que estavam obesas caso tivessem se tornado obesas em uma fase anterior da vida. Ordem decrescente das fases: idade adulta, gravidez, adolescência, acima de 40 anos edepois do casamento (Tabela 2).

Tabela 2. Prevalência e odds da obesidade de acordo com a fase da vida das voluntárias (IM C $>30 \mathrm{~kg} / \mathrm{m}^{2}$, $n=50)$.

\begin{tabular}{lrrr}
\hline & Obesidade & Não obeso & Odds \\
\hline Adolescência & $12(24 \%)$ & $38(76 \%)$ & $32 \%$ \\
Idade adulta & $15(30 \%)$ & $35(70 \%)$ & $42 \%$ \\
Casamento & $1(2 \%)$ & $49(98 \%)$ & $2 \%$ \\
Gravidez & $13(26 \%)$ & $37(74 \%)$ & $35 \%$ \\
$>40$ anos & $4(8 \%)$ & $46(92 \%)$ & $8 \%$ \\
\hline
\end{tabular}

odds $=P \div(1-P)$, sendo $P$ a probabilidade. 0 escoreéo número de voluntárias em determinada condição e entre parênteses a porcentagem em relação ao total.

Tabela 3. Prevalência e odds para os desconfortos emocionais e físicos $(n=50)$.

\begin{tabular}{lrrr}
\hline & Sim & N ão & Odds \\
\hline Ansiedade & $30(60 \%)$ & $20(40 \%)$ & $150 \%$ \\
Depressão & $6(12 \%)$ & $44(88 \%)$ & $13 \%$ \\
Compulsão & $17(34 \%)$ & $33(66 \%)$ & $52 \%$ \\
Distúrbio do sono & $16(32 \%)$ & $34(68 \%)$ & $47 \%$ \\
Dor nas costas & $26(52 \%)$ & $24(48 \%)$ & $108 \%$ \\
Dor no joelho & $17(34 \%)$ & $33(66 \%)$ & $52 \%$
\end{tabular}

odds $=P \div(1-P)$, sendo $P$ a probabilidade. 0 escoreéo número de voluntárias em determinada condição eentre parênteses a porcentagem em relação ao total.
Desconforto emocional/físico

Os principais sintomas de desconforto emocional e físico que foram relatados no exame clínico estão listados na Tabela 3. A maioria era ansiosa, sendo este o distúrbio emocional que mais apareceu. Entre os desconfortos físicos, a lombalgia foi o de maior expressão.

Ingestão de leite e/ou café

No exame clínico, foi feito um levantamento do quanto era ingerido de leite e/ou café por dia pelas voluntárias, procurando conhecer a relação entre um alimento que poderia estar causando relaxamento, no caso o leite, ou ser um estimulante, no caso, o café ${ }^{19}$. A Tabela 4 indica a faixa de volume diário ingerido destas duas bebidas em número de voluntárias. Cinquenta por cento delas ingeriam mais de $500 \mathrm{ml}$ de café/dia contra $24 \%$ das voluntárias que ingeriam este volume deleite.

Dislipidemias, glicemia

e obesidade central

Foram avaliados fatores de risco para doenças metabólicas e DAC, como a concentração de lipídios circulante, hipertensão arterial e obesidade central. 0 valor de corte para glicemia foi de $100 \mathrm{mg} / \mathrm{dL}$, sendo considerado valores maiores que este fator de risco aumentado para o diabetes $^{20}$. H avia importante odds para quase todos os fatores de risco (Tabela 5).
Tabela 4. Ingestão de leite, e ou café em $\mathrm{ml} /$ dia por número de voluntárias em cada faixa de volume da bebida $(n=50)$.

\begin{tabular}{lrr}
\hline \multicolumn{1}{c}{ Volume/bebida } & \multicolumn{1}{c}{ Leite } & \multicolumn{1}{c}{ Café } \\
\hline $0-99$ & $11(22 \%)$ & $14(28 \%)$ \\
$100-199$ & $7(14 \%)$ & $1(2 \%)$ \\
$200-299$ & $11(22 \%)$ & $3(6 \%)$ \\
$300-399$ & $8(16 \%)$ & $7(14 \%)$ \\
$400-499$ & $1(2 \%)$ & $0(0 \%)$ \\
$>500$ & $12(24 \%)$ & $25(50 \%)$ \\
\hline
\end{tabular}

0 escoreéo número de voluntárias em determinada condição eentre parênteses a porcentagem em relação ao total. 
Tabela 5. Prevalência e odds em relação a fatores de risco para diabetes tipo 2 e doenças cardiovasculares.

\begin{tabular}{lcrr}
\hline \multicolumn{1}{c}{ Variáveis } & Sim & Não & Odds \\
\hline Dislipidemicas $^{2}$ & $10(22 \%)$ & $36(78 \%)$ & $61 \%$ \\
Lipídios limítrofes $^{2}$ & $10(22 \%)$ & $36(78 \%)$ & $61 \%$ \\
Glicemia $>100 ~ m g / d L^{20}$ & $12(26 \%)$ & $34(74 \%)$ & $35 \%$ \\
Hipertensas $^{45}$ & $10(20 \%)$ & $39(80 \%)$ & $25 \%$ \\
Obesidade abdominal & $46(92 \%)$ & $4(8 \%)$ & $1150 \%$ \\
(>88 cm) & & & \\
Obesidade central & $26(52 \%)$ & $24(48 \%)$ & $108 \%$ \\
(IAQ) $(>0,90)^{11}$ & & & \\
\hline
\end{tabular}

odds $=P \div(1-P)$, sendo $P$ a probabilidade. 0 escoreéo número de voluntárias em determinada condição e entre parênteses a porcentagem em relação ao total.

Antropometria, $n=50$; Exame de sangue, $n=46$; Pressão arterial, $n=49$.

\section{História familiar de obesidade}

Foi observado o número de familiares obesos de cada voluntária, considerando pai, mãe e irmãos. Trinta e quatro por cento das voluntáriastinham um familiar obeso, outros $34 \%$ tinham dois e $8 \%$ tinham três. No somatório, $76 \%$ tinham um ou mais familiares obesos. Para este grupo de mulheres, havia odds de $316 \%$ deterem um ou mais familiar(es) obeso(s).

\section{Síndrome metabólica}

Quarenta e quatro voluntárias tinham todos os dados para avaliação da síndrome metabólica e 11 (25\%) foram classificadas positivamente, sendo 0 odds de $33 \%$. Para cada faixa do escore dos fatores para síndrome metabólica, o núme ro de voluntárias era (escore dos fatores para síndrome metabólica=n): $0=3 ; 1=15 ; 2=15 ; 3=9$; $4=1 ; 5=1$. 0 resultado do escore dos fatores para síndrome metabólica foi correlacionado $a(0)$ : IAQ $(r=0,27 ; P=0,076)$; I M C ( $r=0,51 ; P=0,000)$; idade $(r=0,40 ; P=0,008)$; circunferência do pescoço $(r=0,37 ; P=0,014)$; prega cutânea do tríceps $(r=0,41 ; P=0,006)$; e prega cutânea do abdômen $(r=0,49 ; P=0,001)$.

\section{Consumo alimentar e gasto energético}

Para análise do consumo calórico, foram escol hidas al eatoriamente sete mulheres a fim dese obter uma amostra. 0 registro alimentar anotado por elas era de uma semana e foram avaliados 28 dias de alimentação, contemplando sempre um dia do final de semana e três durante a semana. Sendo assim, cada voluntária tinha quatro registros. 0 consumo calórico médio aproximado era de $1.900 \pm 300 \mathrm{kcal} / \mathrm{d}$ eo gasto energético de repouso de $1.550 \pm 250 \mathrm{kcal} / \mathrm{d}$.

\section{Bal anço nitrogenado}

Para estimativa do balanço nitrogenado, foram avaliadas catorze amostras de urina sorteadas entre as voluntárias, sendo o resultado de $5 \pm 4 \mathrm{gN} / \mathrm{d}$.

\section{Discussão}

Foi observada influência familiar e comportamental no desenvolvimento da obesidade destas mulheres. Os distúrbios emocionais e físicos tinham prevalência importante entre as voluntárias. A fase adulta e durante a gravidez são períodos da vida em que predomina o sedentarismo e, no caso da maioria destas mulheres, foi quando se tornaram obesas. Existia importante prevalência de dislipidemias, síndromemetabólica e $92 \%$ tinham obesidade abdominal. 0 escore dos fatores para síndrome metabólica foi positivamente correlacionado às variáveis antropométricas e à idade, com exceção do IAQ , que apresentou apenas tendência.

A prevalência para obesidade familiar era de $75 \%$ e havia elevado odds de ter um ou mais deles obeso(s). Resultados similares foram observados quanto à obesidade de adolescentes obesos em Pelotas (RS), associados positivamente aos pais com IM C $>30 \mathrm{~kg} / \mathrm{m}^{22}$. Outros estudos demonstraram relação positiva causa/efeito da obesidade ehiperlipidemia decrianças a obesidade dos pais ${ }^{22}$ e de crianças e adolescentes com a obesidade das mães ${ }^{23}$, comparados aos pais e mães de peso corporal normal respectivamente. Adultos de peso normal, mas com história familiar de obesidade, têm oxidação lipídica pós prandial reduzida ebaixos níveis de insulina. Estes fatores podem predizer precocementeo ganho de peso esão provavelmente determinados geneticamente ${ }^{24}$.

0 desenvolvimento da obesidade destas muIheres foi principalmente na fase adulta e após a gravidez ( $56 \%$ das voluntárias). Outra fase importante para a obesidade destas mulheres foi a adolescência. 0 meio ambiente influenciado pelos aspectos culturais e sociais é importante no desenvolvimento da obesidade, confundindo a relação puramente genética ${ }^{25}$. Fases da vida em que há tendência à redução da atividade física devido a outros interesses, como namorar e fes- 
tas na adolescência, trabalho e família na fase adulta, medo e falta de orientação específica durante a gravidez, entre outros, devem ter contribuído para o elevado odds de se tornarem obesas nestas três fases. As reduções das atividades físicas com a evolução da vida têm demonstrado coincidir com 0 aumento da obesidade ${ }^{26}$.

Outro aspecto relevante observado no presente estudo foi à elevada prevalência de distúrbios emocionais, sendo o odds para ansiedade de 150\%. Distúrbios emocionais podem levar à compensação alimentar hiperfágica, redução da energia pessoal e do estímulo para fazer atividades físicas ${ }^{27}$. Indivíduos com excesso de peso e portadores destes distúrbios apresentam baixa auto-estima e uma visão negativa de seu próprio corpo $^{28}$. Ainda, em outro estudo, a hiperfagia na falta de comportamentos compensatórios era significantemente associada ao aumento da obesidade, sobrepeso, sintomas de desordens alimentares, ansiedade, depressão, ataque de pânico, episódios depressi vos, insônia, menarca precoce e redução da satisfação de viver ${ }^{4}$.

Foi observado queem torno deum terço destas mulheres apresentavam distúrbios relativos ao sono. Isto pode levar ao aumento do consumo de estimulantes para compensar a falta de recuperação física emental, queépropiciada pelo sono de boa qualidade. 0 consumo de café era maior queo deleite, sendo que $70 \%$ delas consumiam mais de $200 \mathrm{ml}$ e $50 \%$, mais de $500 \mathrm{ml}$ de café por dia. 0 al to consumo de caféna, associado à falta de relaxamento, realimenta o estresse físico emental e pode desencadear alguma compensação emocional em busca dealimentos como conforto e não por necessidade nutricional ${ }^{27}$.

Por outro lado, foram estudados o consumo e o gasto energético destas mulheres na tentativa de explicar o sobrepeso ea obesidade. Era observado que elas consumiam em torno de $300 \mathrm{kcal} /$ dia a mais que o GER. É estimado que o GER contribua com até $75 \%$ do gasto energético tota ${ }^{10}$, sendo, portanto, de aproximadamente 2.130 $\mathrm{kcal} /$ dia para estas mulheres. Isso daria um déficit calórico teórico ao redor de $230 \mathrm{kcal} / \mathrm{dia}$, sendo esperado nesta situação que elas estivessem perdendo peso, o quenão ocorria. Provavelmente havia omissão de alimentos do registro alimentar. Heitmann et al. ${ }^{29}$ reforçam que obesos tendem a sonegar no registro alimentar parte dos alimentos ingeridos. Algumas dietas do presente estudo continham apenas entre 400 e $800 \mathrm{kcal} /$ dia, o que não correspondia à condição de sobrepeso e obesidade da maioria delas. Além disso, o balanço nitrogenado era positivo, o que sugere queo aportecalórico e protéico era suficiente para suportar a síntese em vez do catabolismo protéico, que seria esperado em situações de déficit calórico ${ }^{30}$.

Outro aspecto abordado foi a seleção dos macronutrientes que parece influenciar no efeito térmico dos alimentos (ETA). As proteínas podem consumir até $20 \%$ do seu total calórico para serem metabolizadas, os carboidratos, $6-8 \%$ e as gorduras, $2-3 \%{ }^{6}$. Vários estudos têm apontado para o aspecto qualitativo da alimentação rica em lipídios, além do montante calórico total|6,7. Além disso, o poder de saciedade dos macronutrientes do maior para o menor têm a seguinte ordem: proteínas $>$ carboidratos $>$ gorduras ${ }^{31}$. Desta maneira, as gorduras estão relacionadas à obesidade por elevarem o consumo passivo de calori$\mathrm{as}^{32}$, devido à alta palatabilidade e textura características ${ }^{33}$, e terem menor ETA que outros macronutrientes ${ }^{6,7}$. Nesse sentido, como a maioria delas consumiam suas refeições em lanchonetes, que são alimentos ricos em gordura ${ }^{1,5}$, era esperado que isso pudesse explicar, ao menos em parte, 0 sobrepeso e obesidade destas mulheres, 0 que não ocorreu.

Dados similares foram encontrados por Sartorelli ${ }^{34}$, observando que uma população com sobrepeso e obesidade grau I, de São José do Rio Preto, apresentava característica da alimentação ocidental. Têm sido demonstrada mudança nos hábitos alimentares do brasileiro, o que, em parte, poderia ser responsável pelo aumento da obesidade no país. D os consumos alimentares avaliados, apenas $35 \%$ compunham a alimentação típica do brasileiro, à base de arroz, feijão, carne euma salada, que pode ter um aspecto de proteção no desenvolvimento da obesidade ${ }^{35}$. A alimentação típica do brasileiro está associada com mais baixo risco de desenvolver sobrepeso eobesidade comparada à al imentação ocidental, ajustada por modelo logístico para dieta, idade, atividade física de lazer e ocupação $0^{35}$.

Todavia, a média das amostras das dietas analisadas apresentou apenas $24 \%$ das calorias vindas das gorduras, não justificando esta associação em nosso estudo. Dados brasileiros ${ }^{33} \mathrm{e}$ norte-americanos ${ }^{36}$ demonstraram ingestão de $30 \%$ e $40 \%$ das calorias advindas das gorduras em mulheres obesas, respectivamente. Além disso, foi observado que indivíduos com sobrepeso e obesos, consumindo dieta ocidental, tinham consumo alimentar hi percalórico ehiperlipídico (>30\% das calorias) ${ }^{34}$. N ovamente, é possível ter havido sonegação qualitativa das informações das dietas destas voluntárias. 
Entretanto, foi observado que obesos da cidade do Rio deJ aneiro consumiam calorias relativas às gorduras de forma semel hante à observada no presente estudo (mulheres, $28 \%$; homens, $27 \%$ ) ${ }^{9}$. Além disso, ao contrário de outros estudos, Trichopoulou et al. ${ }^{37}$ concluíram que as proteínas mais que os outros macronutrientes estavam associadas positivamente ao IMC e conduziam à obesidade. Já Suen et al. ${ }^{8}$ não observaram diferença no ETA de obesas mórbidas seguindo dieta hipocalórica com diferentes concentrações de macronutrientes. Em outro estudo, o consumo moderado degorduras (33\%) apresentou melhor efeito sobreo perfil lipídico e proteção do desenvolvimento de doenças cardiovasculares, quando comparado ao baixo consumo de gorduras $(18 \%)^{38}$. Não se pode esperar, portanto, que a simples seleção de macronutrientes possa explicar a obesidade destas mulheres. Ainda, além do total calórico dos macronutrientes ingeridos, talvez seja necessário avaliar a sua qualidade, como, por exemplo, a proporção de gorduras poli/monosaturadas, saturadas, trans, ômegas 3, 6 e 97,11,39.

Talvez a característica em comum que mais explique a obesidade destas mulheres seja o sedentarismo. Evidências têm sido acumuladas quanto à importância da preocupação não apenas contra 0 acúmulo de gordura corporal per se, mas antes, devido à pouca atividadefísica praticada pela população ${ }^{40}$, que está associada ao aumento de morbi/mortalidade, independentemente do aumento de peso ${ }^{41}$. No presente estudo, todas as voluntárias eram sedentárias, sugerindo que este fator era determinante na obesidade delas. Vários resultados da literatura têm demonstrado quea falta de atividade física regular talvez seja mais importante no desenvolvimento da obesidade que 0 excesso alimentar ${ }^{42}$. Além disso, a predominância do sedentarismo em obesos favorece o início do ciclo que reduz a energia pessoal e consequentemente prejudica a recuperação e disposição, aumentando o estresse, estimulando a compensação al imentar, o ganho de peso e reduzindo ainda mais a capacidade para atividades físicas ${ }^{27}$.

Todavia, outros estudos não encontraram associação entre o nível de atividade física e obesidade ${ }^{21}$, nem mesmo quanto à ingestão calóri$\mathrm{Ca}^{43}$. M ais uma vez, os resultados indicam que dieta e exercício físico são importantes na gênese e controle da obesidade, mas não podem servir de explicação absoluta para o crescente aumento global da doença.

Uma parcela importante delas apresentava desconforto físico devido a dores osteo-articula- res. Esta condição impede ou prejudica a participação em programas de atividade física. Além do aspecto favorável sobre o metabolismo muscular enos depósitos de lipídios, a atividade física parece exercer importante papel no controle do apetite e na auto-estima dos indivíduos. Ainda, estudos têm demonstrado que parece existir um ponto de ajuste do peso corporal e, quando esteémantido por certo período detempo, tende a ser mantido. Isto justifica, em parte, porque a maioria dos obesos recupera o peso perdido. A atividade física éuma forma de ajustar este ponto para valores mais baixos ${ }^{11,13}$. Portanto, faz-se necessário tratar os problemas relacionados à estrutura musculoesquelética, comuns nos obe$\operatorname{sos}^{13}$, para efetividade dos programas de redução da gordura corporal e melhora do estado emocional destes indivíduos.

Mudanças na ingestão de gordura eatividade física estão positiva e inversamente associadas às mudanças do I M C ${ }^{44}$, I AQ etriglicérides $(\mathrm{Tg})$, respectivamente, melhorando o perfil de risco cardíaco ${ }^{45}$. Embora outros estudos relacionem 0 aumento do IAQ a doenças metabólicas ${ }^{11}$, somente esta variável não teve correlação significativa com a síndrome metabólica. O IM C² e a circunferência do pescoço $0^{46}$ estão associados positivamente ao aumento de fatores de risco e, no presente estudo, foram associados à síndrome metabólica. As medidas de espessura de gordura do tríceps do abdômen e a idade também tiveram associação positiva com a síndrome metabólica. Portanto, algumas medidas simples podem auxiliar no diagnóstico da obesidade e riscos associados e ainda avaliar a evolução de programas de redução de gordura corpora ${ }^{46}$.

A prevalência de dislipidemias no presente estudo era de $22 \%$ e outros $22 \%$ estavam com valores limítrofes. Associado a isso, a grande mai oria delas apresentava obesidade abdominal, queéum fator risco independente para as DAC? Outros estudos mostram associação positiva da obesidade às dislipidemias e a vários fatores de risco para o desenvolvimento de DAC ${ }^{47}$, como os observados no presente estudo.

Por outro lado, os preceitos e normas traçados quanto aos ideais estéticos da sociedade moderna sugerem que o indivíduo deve se adaptar a eles ou ser excluído ${ }^{12}$. Esta condição aumenta ainda mais os desequilíbrios emocionais do obeso, fortalecendo o ciclo virtuoso da doença. Indivíduos que não atingem sucesso em sua empreitada para perda e controle do peso corporal, que é a maioria dos $\operatorname{casos}^{11}$, são vistos como indolentes eindisciplinados ${ }^{13}$, trazendo consigo uma sen- 
sação de fracasso. Como consequência, o indivíduo piora sua auto-estima, diminuindo a importância do controle de peso, tornan do esta meta cada vez mais distante de sua realidade, o que podeter sido a realidade de várias voluntárias do presenteestudo. A incorporação ética deum programa deatividadefísica ealimentação deveriam considerar em primeiro lugar 0 aspecto de saúde e não simplesmente o estético. Para tanto, as re gras deveriam ser flexíveis e adaptadas às necessidades da corporeidade singular, compreendendo as vivências do indivíduo relacionadas ao conhecimento ereflexões sobre seus conflitos internos e ambivalências ${ }^{12}$. No presente estudo, a alta prevalência de distúrbios emocionais caracterizava este desconforto.

Vários fatores interagiram para o desenvolvimento da obesidade destas mulheres, como a ocidentalização dos hábitos, sedentarismo e possivelmente fatores genéticos. Embora estes fatores, tratados isolados ou em conjunto, possam ser associados ao aumento do risco ou agravo de doenças crônicas não transmissíveis, nenhum é necessário, suficiente ou necessário e suficiente para explicá-las. A epidemiologia das doen ças crô- nicas não transmissíveis é incompleta e comple$x a^{48}$, sendo necessária abordagem global e multidisciplinar da vida do indivíduo, reduzindo a dicotomia quantitativo-qualitativa das avaliações de saúde ${ }^{12}$. D eve-se observar a alimentação como um aspecto social e psicológico holístico, constituindo uma dimensão ampla do ser humano e não limitada ao aspecto mecânico e mensurável. Dar ao obeso uma dimensão corpo/espírito enquanto unidade é talvez mais adequado do que apenas avaliar o aspecto quantitativo e punitivo do controle calórico ${ }^{48}$.

Em resumo, a obesidade destas mulheres pareceter múltiplosfatores e consequências, considerando-seinfluências genéticas, fatores ambientais e comportamentais. 0 equilíbrio físico/ emocional com abordagem orgânica e filosófica para alteração de hábitos relacionados ao estilo de vida parece ser necessário no tratamento da obesidade e comorbidades. Este complexo tema pode ser resumido pela observação de Friedman"4: "Em seus esforços para perder peso, indivíduos obesos talvez estejam lutando em um poderoso jogo evolucionário estabelecido em um ambiente drasticamente diferente do atual".

\section{Colaboradores}

CA Fett e WCR Fett participaram da escrita do projeto, coleta e tratamento dos dados e do texto; JS M archini orientou todas as etapas do trabalho eRPP Ribeiro revisou a estatística e texto final. 
Referências

1. Pi-Sunyer $X$. A clinical view of the obesity problem. Science 2003; 299(5608):859-860.

2. National Institute of $\mathrm{H}$ ealth. Third Report of the National Cholesterol Education Program. Detection, Evaluation, and Treatment of $\mathrm{High}$ Blood Cholesterol in Adults (Adult Treatment Panel III). № 01-3670. Washington, D.C.: N ational Institute of H ealth; 2001.

3. Kopelman PG. Obesity as a medical problem. $\mathrm{Na}$ ture 2000; 404(6778):635-643.

4. Reichborn-Kjennerud T, Bulik CM, Sullivan PF, Tambs K, Harris JR. Psychiatric and medical symptoms in binge eating in the absence of compensatory behaviors. O bes Res 2004; 12(9):1445-1454.

5. Chopra M, Galbraith S, Darnton-Hill I. A global response to a global problem: the epidemic of overnutrition. Bull World Health Organ 2002; 80(12):952958.

6. Jéquier $E$. Is fat intake a risk factor for fat gain in children? J Clin Endocrinol Metab 2001; 86(3):980983.

7. Dyck D. Dietary fat intake and weight loss. Can J Appl Physiol 2000; 25(6):495-523.

8. Suen VM M, Silva GA, Tannus AF, Unamuno MRDL. $M$ archini JS. Effect of hypocaloric meals with different macronutrient composition on energy metabolism and lung function in obese women. $\mathrm{Nu}$ trition 2003; 19(9):703-707.

9. Sichieri R. Is fat intake important in the public health control of obesity? Am J Clin Nutri 2000; 72(1):203204.

10. Wilmore JH, Costill DL. Obesity, Diabetes, and Physical activity. In: Physiology of Sport and Exercise. $2^{\text {nd }}$ edition. Champaign: Human Kinetics; 1999. p. 623-687.

11. Rosenbaum M, Leibel RL, Hirsch J. Obesity. N Engl J Med 1997; 337(6):396-407.

12. Carvalho MC, Martins AA. A obesidade como objeto complexo: uma abordagem filosófico-conceitual. Cien Saude Colet 2004; 9(4):1003-1012.

13. Pollock ML, Wilmore JH, Fox III SM. Exercícios na Saúde e na Doença - Avaliação e prescrição para pre venção e reabilitação. Rio de Janeiro: MEDSI; 1986. p. 39-240.

4. Han TS, van Leer EM, Seidell JC, Lean ME. Waist circumference action levels in identification o cardiovascular risk factors: prevalence study in a random sample. BMJ 1995; 311(7017):1401-1405.

15. Munro HN, Fleck A. Analysis of tissues and body fluids for nitrogenous constituents. In: M unro HN, editor. Mammalian protein metabolism. New York and London: Academic Press; 1969. p. 424-462.

16. Weir JB. New methods for calculating metabolic rate with special reference to protein metabolism. J Physiol 1949; 109(1-2):1-9.

17. Pinheiro ABV, Lacerda EMA, Benzecry EH, Gomes MCS, Costa VM. Tabela para avaliação de consumo alimentar em medidas caseiras. 4ạ ed. São Paulo: Atheneu; 2001.

18. Dawson B, Trapp RG. Basic \& Clinical Biostatistics. $2^{\text {nd }}$ ed. New York: Lange Medical Books; 1994. p. 6-183.

19. Fett CA. Ciência da Suplementação Alimentar. Rio de Janeiro: Sprint; 2000.
20. American Diabetes Association. Diagnosis and classification of diabetes mellitus. Diabetes Care 2004; 27(1):S5-S10

21. Neutzling M, Taddei JA, Gigante DP. Risk factors of obesity among Brazilian adolescents: A case-control study. J Adolesc Health 2003; 33(3):143-144.

22. Giugliano R, Carneiro EC. Factors associated with obesity in school children. J Pediatr (Rio J) 2004; 80(1):17-22.

23. M arins VM R, Almeida RM, Pereira RA, Barros M BA. The relationship between parental nutritional status and overweight children/adolescents in Rio de Janeiro, Brazil. Public Health 2004; 118(1):43-49.

24. Giacco R, Clemente G, Busiello L, Lasorella G, Rivieccio AM, Rivellese AA, Riccardi G. Insulin sensitivity is increased and fat oxidation after a highfat meal is reduced in normal-weight healthy men with strong familial predisposition to overweight. Int J Obes Relat M etab Disord 2004; 28(2):342-348.

25. Green J, Waters $E$, Haikerwal A, O'Neill C, Raman $\mathrm{S}$, Booth ML, Gibbons K. Social, cultural and environmental influences on child activity and eating in Australian migrant communities. Child Care Health Dev 2003; 29(6):441-448.

26. Duncan BB, Schmidt MI, Polanczyk CA, Homrich CS, Rosa RS, Achutti AC. Risk factors for non-communicable diseases in a metropolitan area in the south of Brazil. Prevalence and simultaneity. Rev. Saude Publica 1993; 27(1):43-48.

27. Foreyt JP, Goodrick GK. Dieting and weight loss: The energy perspective. Nutri Rev 2001; 59(1):S25S26.

28. Isnard P, Michel G, Frelut ML, Vila G, Falissard B, Naja W, Navarro J, Mouren-Simeoni MC. Int J Eat Disord 2003; 34(2):235-243.

29. Heitmann BL, Lissner L, Osler M. Do we eat less fat, or just report so? Int J Obes Relat M etab Disord 2000; 24(4):435-442.

30. Wolfe RR. Protein supplements and exercises. Am J Clin Nutri 2000; 72(2):551S-557S.

31. Westerterp-Plantenga MS, Lejeune MP, Nijs I, Van Ooijen M, Kovacs EM. High protein intake sustains weight maintenance after body weight loss in humans. Int J Obes Relat M etab Disord 2004; 28(1):5764.

32. Rosado EL, Monteiro JB. Obesidade e a substituição de macronutrientes da dieta. Rev. Nutr. 2001; 14(2):145-152.

33. Pereira LO, Francischi RP, Lancha Júnior AH. Obesidade: hábitos nutricionais, sedentarismo e resistência à insulina. Arq Bras Endocrinol M etab 2003; 47(2):111-127.

34. Sartorelli DS. Estudo nutricional al eatorizado em adultos com sobrepeso em unidade básica de saúde [dissertação]. Ribeirão Preto (SP): Departamento de Medicina Social, Faculdade de M edicina de Ribeirão Preto, Universidade de São Paulo; 2003.

35. Sichieri R. Dietary patterns and their associations with obesity in the Brazilian city of Rio de Janeiro. O bes Res 2002; 10(1):42-48.

36. M ahan LK, Escott-Stump S. Krause: Alimentos, nutrição e dietoterapia. São Paulo: Roca; 1998. 
37. Trichopoulou A, Gnardellis C, Benetou V, Lagiou P, Bamia C, Trichopoulos D. Lipid, protein and carbohydrate intake in relation to body mass index. Euro J Clin Nutri 2002; 56(1):37-43.

38. Pelkman CL, Fishell VK, Maddox DH, Pearson TA, M auger DT, Kris-Etherton PM. Effects of moderate-fat (from monounsaturated fat) and low-fat weight-loss diets on the serum lipid profile in overweight and obese men and women. Am J Clin N utri 2004; 79(2):204-212.

39. Fett CA, Fett WCR. Suplementação e treinamento para redução de peso e melhora da performance em lutador: estudo de caso. Unopar científica ciências biológicas e da saúde 2003/2004; 5/6(1):57-67.

40. Wickelgren I. Obesity: how big a problem? Science 1998; 280(5368):1364-1367.

41. Wei M, Kampert JB, Barlow CE, Nichaman MZ, Gibbons LW, Paffenbarger RS, Blair SN. Relationship between low cardiorespiratory fitness and mortality in normal-weight, overweight, and obese men. JAM A 1999; 282(16):1547-1553.

42. Vincent SD, Pangrazi RP, Raustorp A, Tomson LM, Cuddihy TF. Activity levels and body mass index of children in the United States, Sweden and Australia. Med Sci Sports Exerc 2003; 35(8):1367-1373.

43. Wells JCK, Stanley M, Laidlaw AS, Day JME, Davies PSW. Energy intake in early infancy and childhood fatness. Int J O bes 1998; 22(5):387-392.
44. Paeratakul S, Popkin BM, Keyou G, Adair LS, Stevens J. Changes in diet and physical activity affect the body mass index of Chinese adults. Int J O bes 1998; 22(5):424-431.

45. Matos AC, Ladeia AM. Assessment of cardiovascular risk factors in a rural community in the Brazilian state of Bahia. Arq Bras Cardiol 2003; 81(3):291-302.

46. Fett $C$, Fett W, Fabbro A, Marchini J. Dietary reeducation, exercise program, performance and body indexes associated with risk factors in overweight/ obese women. J Int Soc Sports N utri 2005; 2(2):45-53.

47. Mady C, Fernandes F. IV-Cardiomiopatia da obesidade. Arq Bras Cardiol 2002; 78(1):7-8.

48. Lessa I. Doenças crônicas não transmissíveis no Brasil: um desafio para a complexa tarefa da vigilância. Cien Saude Colet 2004; 9(4):931-943.

49. Friedman JM. A war on obesity, not the obese. Science 2003; 299(5608):856-858.

Artigo apresentado em 12/03/2006

Aprovado em 27/06/2007

Versão final apresentada em 09/09/2007 